J Venom Anim Toxins incl Trop Dis, 2019 25: e147618

\author{
The Journal of Venomous Animals and \\ Toxins including Tropical Diseases \\ ISSN 1678-9199 \\ Journal homepage www.jvat.org
}

\title{
BjussuLAAO-II induces cytotoxicity and alters DNA methylation of cell-cycle genes in monocultured/co-cultured HepG2 cells
}

\author{
Ana Rita Thomazela Machado', Alexandre Ferro Aissa', Diego Luis Ribeiro², Rui Seabra Ferreira Jr. ${ }^{3}$, \\ Suely Vilela Sampaio ${ }^{1}$, Lusânia Maria Greggi Antunes ${ }^{1, *}$ (D) \\ 1 Department of Clinical Analysis, Toxicology and Food Sciences, School of Pharmaceutical Sciences of Ribeirão Preto, \\ University of São Paulo - USP, Ribeirão Preto, SP, Brazil. \\ ${ }^{2}$ Department of Genetics, Ribeirão Preto Medical School, University of São Paulo - USP, Ribeirão Preto, SP, Brazil. \\ ${ }^{3}$ Center for the Study of Venoms and Venomous Animals (CEVAP), São Paulo State University - UNESP, Botucatu, SP, Brazil.
}

\section{Article Info \\ Keywords:}

snake venom

epigenetics

GADD45A

CCND1

CDKN1A

\begin{abstract}
Background: The use of animal venoms and their toxins as material sources for biotechnological applications has received much attention from the pharmaceutical industry. L-amino acid oxidases from snake venoms (SV-LAAOs) have demonstrated innumerous biological effects and pharmacological potential against different cancer types. Hepatocellular carcinoma has increased worldwide, and the aberrant DNA methylation of liver cells is a common mechanism to promote hepatic tumorigenesis. Moreover, tumor microenvironment plays a major role in neoplastic transformation. To elucidate the molecular mechanisms responsible for the cytotoxic effects of SV-LAAO in human cancer cells, this study aimed to evaluate the cytotoxicity and the alterations in DNA methylation profiler in the promoter regions of cell-cycle genes induced by BjussuLAAO-II, an LAAO from Bothrops jaracussu venom, in human hepatocellular carcinoma (HepG2) cells in monoculture and co-culture with endothelial (HUVEC) cells. Methods: BjussuLAAO-II concentrations were $0.25,0.50,1.00$ and $5.00 \mu \mathrm{g} / \mathrm{mL}$. Cell viability was assessed by MTT assay and DNA methylation of the promoter regions of 22 cell-cycle genes by EpiTect Methyl II PCR array.

Results: BjussuLAAO-II decreased the cell viability of HepG2 cells in monoculture at all concentrations tested. In co-culture, 1.00 and $5.00 \mu \mathrm{g} / \mathrm{mL}$ induced cytotoxicity $(p<0.05)$. BjussuLAAO-II increased the methylation of CCND1 and decreased the methylation of CDKN1A in monoculture and GADD45A in both cell-culture models $(p<0.05)$.

Conclusion: Data showed BjussuLAAO-II induced cytotoxicity and altered DNA methylation of the promoter regions of cell-cycle genes in HepG2 cells in monoculture and co-culture models. We suggested the analysis of DNA methylation profile of GADD45A as a potential biomarker of the cell cycle effects of BjussuLAAO-II in cancer cells. The tumor microenvironment should be considered to comprise part of biotechnological strategies during the development of snake-toxin-based novel drugs.
\end{abstract}

* Correspondence: 


\section{Background}

The use of animal venoms and their toxins as natural sources of material for biotechnological application has received much attention from the pharmaceutical industry and experts in the field of applied research. Since the development of captopril, the first drug derived from a bradykinin-potentiating peptide from Bothrops jararaca, to the disintegrins that have displayed potent activity against certain types of cancers, the components of snake venom (SV) have shown great potential for the development of new candidate drugs [1-3]. SV is probably the most complex venom and consists of metalloproteinases, disintegrins, phospholipase A2, lectins, and L-amino acid oxidases (LAAOs) [4].

LAAOs constitute $1-9 \%$ of the total venom protein and are responsible for the yellowish coloration of the venom [5]. Many studies of biological characterization of L-amino acid oxidase isolated from SVs (SV-LAAOs) have already demonstrated innumerous biological effects and pharmacological potential of these proteins [6, 7]. In this sense, SV-LAAOs could be used for the development of new and more effective drugs against different types of cancer [8].

Cancer is one of the leading causes of death in the world, and there is an urgent need to find better treatments [9]. Hepatocellular carcinoma (HCC) is the most common type of primary cancer of the liver, ranked as the fifth most frequent cancer and the third greatest cause of cancer mortality in the world [10]. HCC is often diagnosed late in individuals with severe hepatic impairment, thus limiting the options for chemotherapy and adjuvant therapies. Also, the lack of early detection markers and drug resistance may contribute to the high mortality rate in HCC [10]. Moreover, increasing evidence suggests that the development of HCC is the result of a multi-step process involving many genetic and epigenetic abnormalities that result in abnormal gene expression [10, 11].

HCC tumors display distinct DNA methylation signatures associated with risk factors, tumor stage, degree of differentiation, and survival after antineoplastic therapy [11]. In the HCC genome, hypomethylation affects the structural-nuclear function by promoting chromosomal and genomic instability, whereas hypermethylation is often associated with the silencing of tumor-suppressor genes. Together, these processes determine hyperactivation of progressive steps in hepatocarcinogenesis [12].

Aberrant DNA methylation of Cytosine-phosphate-Guanine $(\mathrm{CpG})$ islands is thought to be one of the most common epigenetic change in cancer, usually resulting in impairment of gene expression. In the mammalian genome, DNA methylation occurs by the covalent addition of a methyl $(-\mathrm{CH} 3)$ group to cytosine residues in $\mathrm{CpG}$ dinucleotides. Nearly $70 \%$ of the gene promoters annotated in the human genome are characterized by high CpG content. Methylation of DNA in CpG islands located in gene promoter regions is associated with differential gene expression. The altered epigenetic state may lead to deregulation of cellular processes, such as proliferation, transformation, and antiapoptotic mechanisms, which promote tumorigenesis [11, 13]. DNA methylation can mediate gene silencing directly by inhibiting the binding of methylation-dependent transcriptional activators or indirectly by altering the affinity of the proteins involved in chromatin remodeling. In cancer cells, the loss of DNA methylation mainly affects the repetitive genomic elements and gene bodies, whereas hypermethylation occurs mainly in the promoters of tumor suppressor genes [10].

Recent studies have reported that the tumor microenvironment plays a major role in neoplastic transformation, whereas the presence of endothelial cells also correlates with induction of chemosensitization and chemoresistance [14-16]. In order to better understand the molecular mechanisms responsible for the cytotoxic effects of SV-LAAO on human cancer cells in vitro, the purpose of this study was to evaluate the cytotoxicity and the alterations in DNA methylation profile of cell cycle genes induced by BjussuLAAO-II, an LAAO isolated from Bothrops jararacussu snake venom, in human hepatocellular carcinoma (HepG2) cells in monoculture and in co-culture with an endothelial cell line (HUVEC).

\section{Methods}

\section{Toxin}

BjussuLAAO-II was isolated from Bothrops jararacussu snake venom according to the procedure described by Carone $e t$ al. [17]. The toxin is an acidic enzyme that exhibits high enzymatic activity $(4,884.53 \mathrm{U} / \mathrm{mg} / \mathrm{min})$, has isoelectric point of 3.9 and molecular mass of $60.36 \mathrm{kDa}$, and represents $0.3 \%$ of the venom proteins. Before performing the biological assays, LAAO enzymatic activity was determined by a spectrophotometric assay using L-leucine as a substrate [18]. The isolated and purified protein was stored at $4^{\circ} \mathrm{C}$. The vehicle employed to dilute the protein was phosphate buffered saline (PBS, $\mathrm{pH}$ 7.4).

\section{Cell lines and culture conditions}

Human hepatocarcinoma cells (HepG2 - catalog \#HB8065) and human umbilical-vein endothelial cells (HUVEC - catalog \#CRL-1730) were obtained from the American Type Culture Collection (ATCC, Manassas, Virginia, USA). The cells were maintained in RPMI 1640 medium supplemented with $10 \%$ FBS, $1 \%$ antibiotic-antimycotic solution $(5 \mathrm{mg} / \mathrm{mL}$ penicillin, $5 \mathrm{mg} / \mathrm{mL}$ streptomycin, and $10 \mathrm{mg} / \mathrm{mL}$ neomycin), and $0.024 \%$ (w/v) $\mathrm{NaHCO}_{3}$, in a $\mathrm{CO}_{2}$ incubator with $5 \%$ atmosphere, at $37^{\circ} \mathrm{C}$ and relative humidity of $96 \%$. The media were changed every 2-3 days; when the cultures had reached confluency, the cells were washed twice in PBS, detached with Trypsin/EDTA $(0.25 \%)$, centrifuged at $174 \mathrm{x} g$ for $5 \mathrm{~min}$ and sub-cultured. All the experiments were conducted between the third and the eighth cell passage and they were cultured as reported by BalPrice and Coecke [19].

\section{Co-culture system}

Thincert $^{\mathrm{mw}}$ (Greiner Bio-one, Kremsmünster, Austria) cell-culture inserts with $0.4 \mu \mathrm{m}$ porous polycarbonate membranes in 6 -well 
plates were used in cellular co-culture systems. HepG2 cells $\left(2 \times 10^{5}\right.$ cells/well $)$ were grown adhering to the bottom of the well whereas HUVEC cells $\left(1 \times 10^{4}\right.$ cells/well $)$ were grown in the upper compartment [20-23]. The Millicell ERS* volt-ohm meter (Merck-Millipore, Burlington, Massachusetts, USA) was employed to monitor electrical resistivity of HUVEC cells. The inserts whose transepithelial electrical resistance was greater than or equal to $750 \Omega / \mathrm{cm}^{2}$ were considered confluent; when this value was reached, HepG2 cells were seeded underneath the well in co-culture plates. Experiments in co-culture systems followed the same protocols described for monoculture systems.

\section{MTT assay}

Cell viability was determined using the MTT assay, as reported by Mosmann [24]. In monoculture systems, HepG2 and HUVEC $\left(1 \times 10^{4}\right.$ cells/well $)$ were seeded in 96 -well plates. In co-culture systems, 6-well plates were used and HepG2 were seeded in the lower $\left(4 \times 10^{5}\right.$ cells/well $)$ and HUVEC $\left(1 \times 10^{4}\right.$ cells/well $)$ was placed in upper compartments. In both systems, cells were incubated for $24 \mathrm{~h}$ and treated with BjussuLAAO-II (0.25; $0.50 ; 1.00$ and $5.00 \mu \mathrm{g} / \mathrm{mL}$ ), PBS (negative control) or methyl methanesulfonate (MMS; CAS: 66-27-3; positive control) for $72 \mathrm{~h}$. The supernatant was removed, and $0.2 \mathrm{~mL}$ or $3.0 \mathrm{~mL}$ of MTT solution $(5 \mathrm{mg} / \mathrm{mL}$ ) were added to the wells in monoand co-culture systems, respectively. After $3 \mathrm{~h}$ of incubation, the supernatant was replaced by equivalent volumes of DMSO (Sigma Aldrich, St. Louis, Missouri, USA) and absorbance was recorded in a spectrophotometer (Biotek Elx800 - Winooski, VT, USA) set at $570 \mathrm{~nm}$. Absorbance values of the negative control were defined as constituting $100 \%$ cell viability, and the results were expressed as a percentage (\%) of viable cells.

\section{EpiTect methyl qPCR array analysis}

HepG 2 cells in mono- and co-culture were cultivated as described in the MTT assay. The methylation of the promoter region of 22 cell-cycle genes was analyzed using EpiTect Methyl II PCR Array Human Cell Cycle Signature PCR Array (EAHS-201ZC-2, Qiagen, Hilden, Germany) following the manufacturer's protocol. Genes are related to the G1 phase (CCND1, CCNE1, CDK4, CDKN1B), S Phase and Replication (MCM2, MCM4), G2 Phase \& G2/M Transition (CCNB1, CDK5RAP1, CKS1B), M Phase (CCNF, MRE11A, RAD51), Cell-Cycle Checkpoint \& Cell-Cycle Arrest (ATM, BRCA1, BRCA2, CDK2, CDKN1A, CDKN1B, CHEK1, GADD45A, RAD9A, TP53) and Regulation of Cell Cycle (ATM, BRCA1, BRCA2, CCNB1, CCND1, CCNE1, CCNF, CDK2, CDK4, CDKN1A, CDKN1B (p27KIP1), CKS1B, GADD45A, $R A D 9 A, R B L 1$, TP53). This assay is based on the digestion of unmethylated and methylated DNA, using methylationsensitive and methylation-dependent restriction enzymes. Genomic DNA from HepG2 treated for $72 \mathrm{~h}$ with $0.25 \mu \mathrm{g} / \mathrm{mL}$ of BjussuLAAO-II was isolated using the DNeasy Blood and Tissue Kit (Cat \#69504, Qiagen, Hilden, Germany) according to the manufacturer's protocol. The genomic DNA was quantified by spectrophotometry (NanoDrop 2000C; Thermo Scientific, San Jose, CA, USA) with all samples showing a A260/A280 ratio higher than 1.8. The digestion was performed employing the EpiTect Methyl II DNA Restriction Kit (Qiagen, Hilden, Germany). A reaction mix without enzymes was prepared from $1 \mu \mathrm{g}$ genomic DNA, $26 \mu \mathrm{L}$ of $5 \times$ Restriction Digestion Buffer, and RNase-DNase-free water to make the final volume $120 \mu \mathrm{L}$. Four digestion reactions - Mo (no enzyme - mock digestion), Ms (methylation sensitive), Md (methylation-dependent), and Msd (double) were set up. The samples were incubated at $37^{\circ} \mathrm{C}$ for $6 \mathrm{~h}$. Then quantitative PCR (qPCR) was performed using the RT2 SYBR Green ROX qPCR Master Mix (Qiagen, Hilden, Germany) according to the manufacturer's recommendations in a Step One Plus Real-Time PCR System (Applied Biosystems, Foster City, CA, USA). Threshold cycle (Ct) values were utilized to calculate the percentages of methylated $(\mathrm{M})$ and unmethylated (UM) DNA, using a quantization algorithm provided by the manufacturer to normalize the amount of DNA in each digestion against the total amount of input DNA in the mock digestion, using the Excel macro-spreadsheet supplied by the manufacturer (Qiagen, Hilden, Germany) [25-27].

\section{Statistical analysis}

All the results were expressed as the mean \pm standard deviation (SD) of triplicates $(n=3)$. Kolmogorov-Smirnov test was employed to test the normality, and experimental data were analyzed using one-way analysis of variance (ANOVA) followed by the Tukey's test in MTT assay and Dunnett's test in DNA methylation assay. Data were statistically analyzed using the software GraphPad Prism 5.00 for Windows (GraphPad Software, La Jolla, CA, USA) and statistical significance was considered at $p<0.05$.

\section{Results}

\section{BjussuLAAO-II is cytotoxic towards HepG2 and HUVEC cells}

At all concentrations tested (in the range from 0.25 to $5.00 \mu \mathrm{g}$ / $\mathrm{mL}$ ), BjussuLAAO-II decreased the MTT reduction capacity in HepG2 (Fig. 1A) and HUVEC (Fig. 1B) cells in monocultures. In HepG 2 co-culture, only the 1.00 and $5.00 \mu \mathrm{g} / \mathrm{mL}$ concentrations significantly decreased the cell viability (Fig. 1C) when compared to the negative control. MMS $(300 \mu \mathrm{M})$, used as positive control, was effective at decreasing cell viability in HepG2 and HUVEC in both monoculture and in co-culture.

\section{BjussuLAAO-II altered DNA methylation in the promoter region of cell-cycle genes}

Since $0.25 \mu \mathrm{g} / \mathrm{mL}$ of BjussuLAAO-II was not cytotoxic in coculture, this concentration was selected to assess the effect of the toxin on the DNA methylation of the promoter region of 22 cell-cycle genes in HepG2 cells in monoculture and coculture (Fig. 2 and Fig. 3). As can be seen in Fig. 3, after $72 \mathrm{~h}$ of exposure, of the total of 22 cell-cycle genes, six were statistically 
(A)

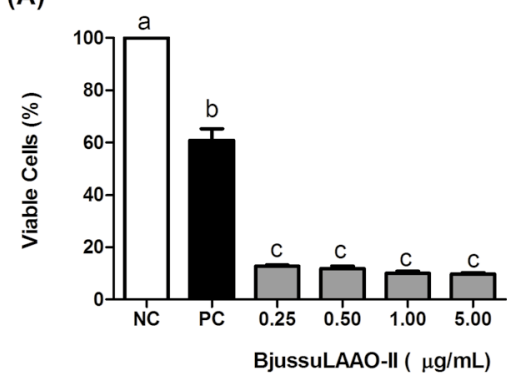

(B)

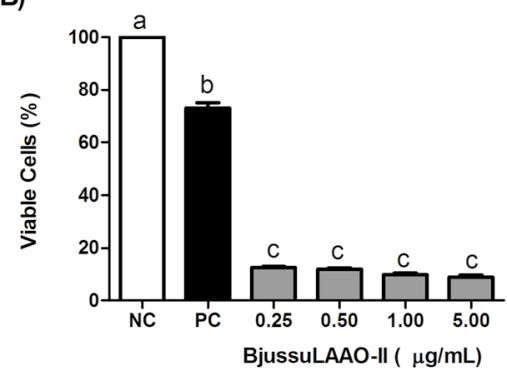

(C)

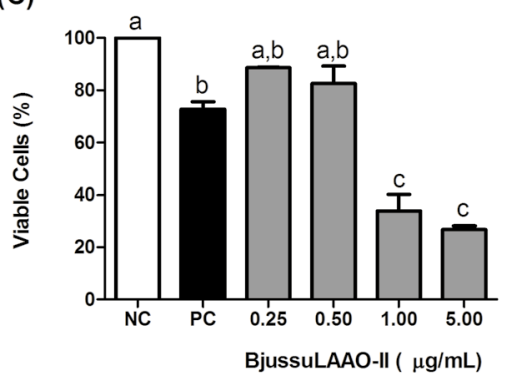

Figure 1 Cell viability of HepG2 and HUVEC cells treated with BjussuLAAO-ll for $72 \mathrm{~h}$. A: HepG2 cells in monoculture. B: HUVEC cells in monoculture. C: HepG2 cells in co-culture. NC: Negative Control (PBS, pH 7.4). PC: Positive Control (300 $\mu M$ MMS). Percentage (\%) of cell viability, assessed by the MTT assay, was calculated for the NC and expressed as mean \pm SD $(n=3)$. Bars not sharing the same letter are significantly different from each other $(p<0.05$; ANOVA followed by the Tukey test).

altered in monoculture treatment and two genes were altered in co-culture when compared to the respective negative control. As to DNA methylation in monoculture, CCND1 showed an increase while CDKN1A, GADD45A, MCM4, RAD51 and TP53 presented a decrease. In co-culture, BjussuLAAO-II decreased the DNA methylation of GADD45A and increased the DNA methylation of CCNB1 (Fig. 3). To select the genes with a more biologically relevant alteration, a methylation change threshold of $>20 \%$ was applied in non-treated cells [28]. CCND1 showed a methylation decrease from $100 \%$ in negative control to $72 \%$ in BjussuLAAO-II-treated cells in monoculture. CDKN1 produced methylation of $49 \%$ in negative control and $80 \%$ in monoculture, i.e., a 31\% increase of DNA methylation induced by BjussuLAAO-II. GADD $45 A$ was the gene most influenced by the BjussuLAAO-II since in monoculture the DNA methylation of its promoter region increased from $30 \%$ in negative control to $80 \%$ in monoculture and $50 \%$ in co-culture.

\section{Discussion}

Biotechnological strategies are needed for the development of new drug candidates to improve the treatment of cancer. The search for antineoplastic drugs obtained from natural biological resources is global. Venoms and toxins are rich in several bioactive substances, which makes them an excellent source for the discovery of new drugs. Thus, it is important to assess the anticancer effects of animal toxins on human cancer cells to decide the best candidates for potential clinical use. In vivo and in vitro studies have demonstrated that animal toxins, either isolated or in raw venom, have inhibitory effects against cancer cells $[29,30]$. Many SV-LAAOs isolated from different snake species have been described as capable of inducing cell death in diverse cell lines by reducing the cell viability measured by the MTT assay. Previous studies have shown that cytotoxicity is an important function of these enzymes [31, 32].

$\mathrm{OH}-\mathrm{LAAO}$, isolated from the Ophiophagus hannah snake, has demonstrated antiproliferative activity in human breast cancer cells (MCF-7) and in human lung adenocarcinoma cells (A549) after $72 \mathrm{~h}$ of treatment [33]. This antiproliferative effect is highly selective, with $\mathrm{IC}_{50}$ values being 3 - and 4 -fold higher in non- tumor cells of human mammary gland (184B5) and bronchial epithelium (NL20), respectively [33]. BjussuLAAO-II after $72 \mathrm{~h}$ of treatment also reduced cell viability in HepG2. Despite reports that claimed selective cytotoxicity of SV-LAAO in cancer cell lines, LAAOs from some species have exhibited cytotoxic effects on non-tumor cells as well [5]. Similar results are reported in the present study from BjussuLAAO-II since the toxin reduced the viability of the non-tumor HUVEC in the MTT assay. In a previous study, BjussuLAAO-II was tested against human colorectal adenocarcinoma cells (Caco-2) in a $24 \mathrm{~h}$ treatment and also demonstrated the reduction of cell viability [34].

It has been proposed that the specific effects of SV-LAAOS on biological systems are due to the generation of hydrogen peroxide $[6,31,35]$. However, the molecular mechanisms by which SV-LAAOs induce cytotoxicity and apoptosis in cancer cells are not fully understood.

There are few reported studies in the literature on the DNA methylation changes or histone modification in response to snake venoms or snake toxins in vitro. Wu et al. [36] found that melittin, a major component of bee venom, can inhibit human hepatocarcinoma (SMMC-7721) cell proliferation due to delay in cell-cycle progression. The results showed that melittin decreases the methylation of $\mathrm{PTCH} 1$, which is important for tumorigenesis, resulting in the increased expression of its protein in vitro. In order to provide information about the involvement of epigenetic regulation in a rat model of bee venom-induced inflammatory pain, Yang et al. [37] investigated selective class I histone deacetylase inhibitors (HDACIs) in rats inflamed by subcutaneous injection of whole venom from Apis millifera. Changes in histone deacetylase 1 (HDAC1) and 2 (HDAC2) expression patterns were observed in intact rat lumbar spinal cord after bee venom injection and treatment with HDCA inhibitors.

DNA methylation of CpG islands is the best known important epigenetic mechanism in cancer progression. Aberrant DNA methylation of $\mathrm{CpG}$ islands is thought to be one of the most common changes in the silencing of cell-cycle genes in various tumor types [38]. Gene regulation is controlled by different mechanisms, such as epigenetic processes, that influence several essential pathways and stages of carcinogenesis including tumor initiation and progression. Deregulation of the cell cycle is a 

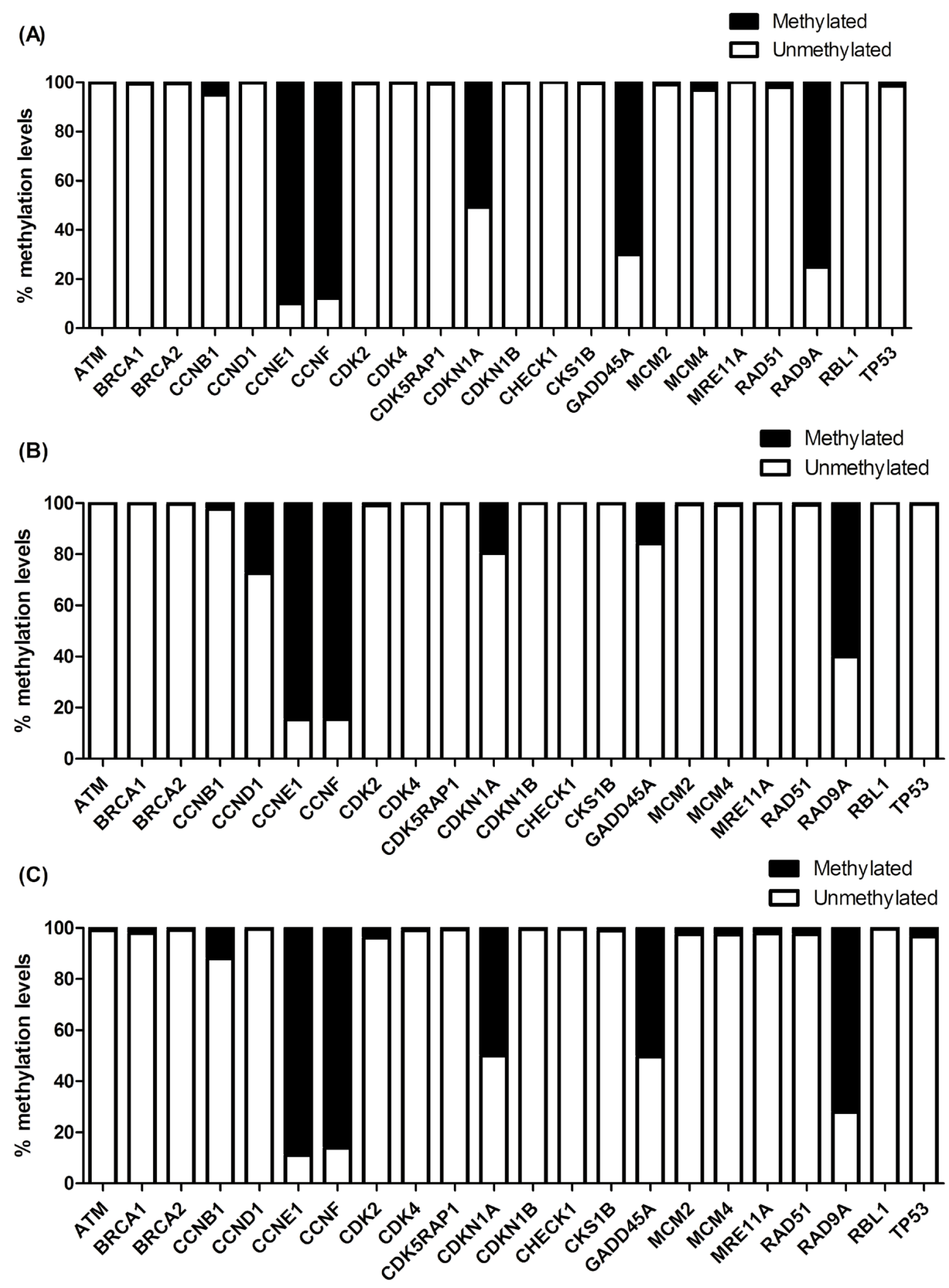

Figure 2 DNA methylation profile in promoter region of cell cycle genes after 72h of BjussuLAAO-II treatment. A: HepG2 cells in monoculture treated with PBS (pH 7.4), used as negative control. B: HepG2 cells in monoculture treated with BjussuLAAO-II (0.25 $\mu g / \mathrm{Ml})$. C: HepG2 cells in co-culture treated with BjussuLAAO-II $(0.25 \mu \mathrm{g} / \mathrm{mL})$.

common characteristic of human cancer while cell-cycle regulatory proteins are critical determinants of malignancy progression. Genes controlling G1, S, G2 and M phases of the cell cycle including cyclin-dependent kinases (CDKs), cyclins activating CDKs and cyclin-dependent kinase inhibitors (CDKIs) - are crucial for the control of mammalian cell proliferation [38, 39].
The genes most affected by BjussuLAAO-II, with an alteration higher than $20 \%$ as compared with non-treated cells, were CDKN1A (Cyclin-Dependent Kinase Inhibitor 1A), CCND1 (Cyclin D1), and GADD45A (Growth Arrest and DNA-DamageInducible $45 \mathrm{Alpha}$ ). CDKN1A controls the proliferation, differentiation and tumorigenesis of many cancer cell types 


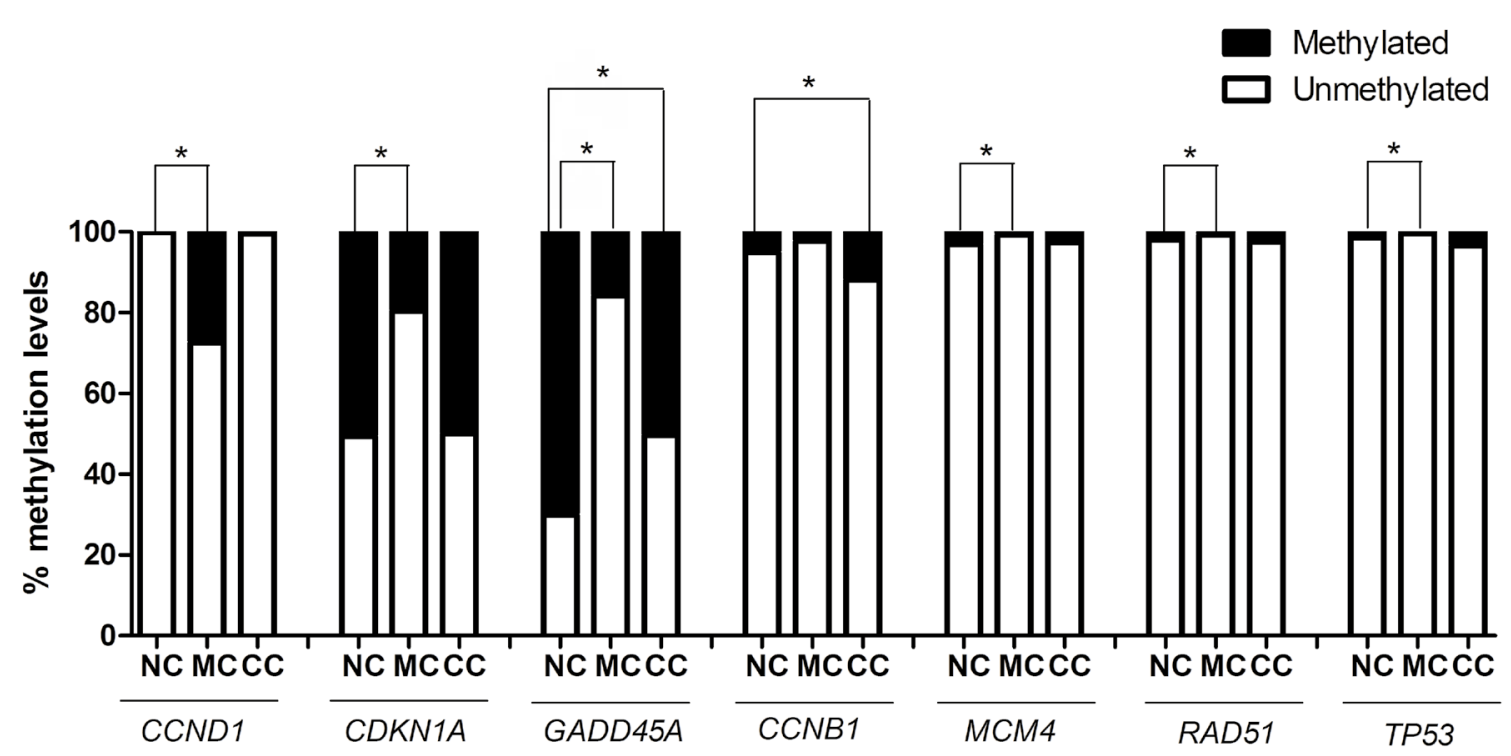

Figure 3 Genes with significant changes in DNA methylation in promoter region after $72 \mathrm{~h}$ of BjussuLAAO-II treatment. NC: HepG2 cells in monoculture treated with PBS (pH 7.4), used as negative control. MC: HepG2 cells in monoculture treated with BjussuLAAO-II (0.25 $\mu$ g/MI). CC: HepG2 cells in co-culture treated with BjussuLAAO-II $(0.25 \mu \mathrm{g} / \mathrm{mL})$. *Values with significant difference $(p<0.05$; ANOVA followed by Dunnett's test) compared to NC.

and plays a key role in the p53-mediated cell cycle arrest [40]. Generally, CDKN1A has been observed hypermethylated and down-regulated in HCC tissues, which might promote cellcycle progression with consequent uncontrolled proliferation of liver cells with pre-cancerous lesions thereby contributing to oncogenesis [10, 41, 42]. BjussuLAAO-II decreased the methylation of the promoter region of this gene, which can return to the normal epigenetic status, increasing gene expression and stopping the cell cycle. In fact, a decrease was observed in cell viability as measured by the MTT assay in HepG2 cells. Similar effects were reported by Obata et al. [40], who found that hypomethylation of the CDKN1A promoter region resulted in cell-cycle arrest in intestinal regulatory T cells in vitro.

CCND1 is a key cell-cycle regulator and one of the most important oncogenes, being overexpressed in several human cancers. CCND1 forms a complex with CDK4 to control cellcycle progression through the G1 phase and to promote the cell-cycle transition into the $S$ phase by activation of the CCNE1/ CDK2 complex [43, 44]. Overexpression of CCND1 disrupts the normal cell cycle, possibly promoting the development and progression of cancer [43]. However, it does not exert tumorigenic activity by itself but rather cooperates with other altered oncogenes and tumor suppressors to induce and govern neoplastic transformation [44]. Lin et al. [45] demonstrated that Protease Serine 3 (PRSS3) exerts tumor-suppressive functions in human HCC by inducing G1/S cell-cycle arrest and suppressing the complexes of CCND1/CDK4. The results reported herein suggest that BjussuLAAO-II may also induce cell-cycle arrest by increasing the methylation of CCND1.

GADD $45 A$ is a tumor suppressor gene that has cell-typespecific roles in cellular stress, coordinating DNA repair and demethylation, cell-cycle arrest and pro-apoptotic or prosurvival responses [46]. An upregulation of GADD45A has been shown to protect against DNA damage and uncontrolled cellular proliferation [47]. GADD45A family members are multi-faceted nuclear factors implicated in active DNA demethylation, apart from maintenance of genomic stability, DNA repair and suppression of cell growth in vertebrates [48]. The hypomethylation of the GADD45A gene caused by BjussuLAAO-II may increase its expression leading to the demethylation of tumor suppressor genes, increasing their expression and consequently, decreasing cell proliferation in monoculture. There was also a decrease in DNA methylation of the GADD45A in HepG2 cells in co-culture, evidencing that BjussuLAAO-II is able to alter the methylation pattern of this gene even in the tumor microenvironment. Serum GADD45A methylation was previously indicated as a useful biomarker to distinguish benign versus malignant prostate cancer [49]. Moreover, we suggested that GADD $45 A$ may serve as a suitable biomarker of the effects of BjussuLAAO-II on DNA methylation, since this gene is indicated to distinguish between benign versus malignant prostate disease in patients.

The tumor microenvironment plays an essential role in proliferation, migration, survival and drug resistance in human tumors [50] and in cell culture [51]. For example, hepatocyte growth factor, one of the important growth factors in the tumor microenvironment, upregulated the expression of DNA Methyltransferase 1 (DNMT1) in HepG2 and noncancer human liver cells (HL-7702) cells and the overexpression of DNMT1 in HCC patients correlated with the malignant potential and poor prognosis [52]. For this reason, the use of co-cultures, such as HepG2 co-cultured with endothelial cells, is an important tool to understand the tumor microenvironment in hepatocarcinoma development [16]. As can be observed both in the MTT assay and in the DNA methylation in the promoter region of cell cycle genes, the tumor microenvironment plays an important role in the cellular response to BjussuLAAO-II treatment. 


\section{Conclusion}

Our findings may provide new insights into the mechanisms by which toxin snake venom is cytotoxic against hepatocellular carcinoma cells. Specifically, BjussuLAAO-II may exert its cytotoxicity in HepG2 cells by decreasing the methylation of tumor-suppressor genes including CDKN1A and GADD45A and increasing the methylation of oncogenes such as CCND1. We suggest the analysis of the DNA methylation profile of GADD $45 A$ as a potential biomarker of the cell-cycle effects of BjussuLAAO-II in cancer cells. Furthermore, cell viability and DNA methylation changes were dependent on the cell-culture model. For this reason, we proposed that the role of the tumor microenvironment should be considered in biotechnological studies investigating novel chemotherapeutic drugs rather than only in vitro monoculture assays.

\section{Acknowledgements}

The authors are grateful to Mr. Sante Emmanuel Imai Carone from the Laboratory of Toxinology at the School of Pharmaceutical Sciences of Ribeirão Preto, University of São Paulo, Brazil (FCFRP-USP), for isolating BjussuLAAO-II; and to Dr. Regislaine Valéria Burim and Ms. Joana D’Arc Castania Darin from the Department of Clinical Analysis, Toxicology and Food Sciences at FCFRP-USP for their technical assistance.

\section{Abbreviations}

BjussuLAAO-II: LAAO from Bothrops jararacussu venom; CCND1: Cyclin D1; CDKN1A: Cyclin-Dependent Kinase Inhibitor 1A; $C p G$ : Cytosine-phosphate-Guanine island; DNMT1: DNA Methyltransferase-1; GADD45A: Growth Arrest And DNA-Damage-Inducible 45 Alpha; HCC: Hepatocellular carcinoma; HepG2: Hepatocarcinoma cells; HDAC: Histone deacetylase inhibitors; HUVEC: Human umbilical vein endothelial cells; LAAO: L-amino acid oxidase; MMS: Methyl methanesulfonate; MTT: 3-(4,5-dimethylthiazol-2-yl)-2,5diphenyltetrazolium bromide; Mo: No enzyme digestion; Ms: Methylation-sensitive enzyme digestion; Md: Methylationdependent enzyme digestion; Msd: Double-enzyme digestion; PBS: Phosphate Buffered Saline; SV: Snake Venom; SV-LAAO: LAAO from snake venom.

\section{Availability of data and material}

Not applicable.

\section{Funding}

This publication was supported in part by the Coordination for the Improvement of Higher Education Personnel (CAPES) through "Programa Editoração CAPES" - call No. 3/2016, grant No. 0722/2017, record No. 88881.142062/2017-01 and by the National Council for Scientific and Technological Development (CNPq) and Coordination for the Improvement of Higher Education Personnel (CAPES) through "Programa Editorial
CNPq/CAPES” call No. 18/2018, grant No. 404770/2018-5. The authors thank the São Paulo Research Foundation (FAPESP, Brazil, grant \#2011/23236-4) and the Research Support Center on Animal Toxins (NAP-TOXAN-USP, Brazil) for funding this research. LMG Antunes, RS Ferreira Jr, and SV Sampaio received research productivity fellowships from the CNPq. The authors are also grateful to CAPES for the scholarship to ARTM (Financing Code 001) and PRONEX, for the StepOnePlus ${ }^{\text {тM }}$ equipment (USP/FUSP/FINEP convention n. 01.09.0447.00/ CT-INFRA2008 subproject 228.524).

\section{Competing interests}

The authors declare that they have no competing interests.

\section{Authors' contributions}

ARTM was responsible for project development, designed the experimental approaches, interpreted the data, performed the experiments and drafted the manuscript. DLR and AFA participated in the designed experiment, interpreted data and drafted the manuscript. RSFJR and SSV provided the toxin and drafted the manuscript. LMGA coordinated and designed all the experiments, analyzed and interpreted the data, and was a major contributor in writing the manuscript. All authors read and approved the final manuscript.

\section{Ethics approval and consent to participate}

Not applicable.

\section{Consent for publication}

Not applicable.

\section{References}

1. Waheed H, Moin SF, Choudhary MI. Snake venom: from deadly toxins to life-saving therapeutics. Curr Med Chem. 2017;24(17):1874-91.

2. O'Shea JC, Tcheng JE. Eptifibatide: a potent inhibitor of the platelet receptor integrin glycoprotein Ilb/Illa. Expert Opin Pharmacother. 2002;3(8):1199. 210.

3. Marcinkiewicz C. Functional characteristic of snake venom disintegrins: potential therapeutic implication. Curr Pharm Des. 2005;11(7):815-27.

4. Dhananjaya BL, Sivashankari PR. Snake venom derived molecules in tumor angiogenesis and its application in cancer therapy; an overview. Curr Top Med Chem. 2015;15(7):649-57.

5. Tan KK, Bay BH, Gopalakrishnakone P. L-amino acid oxidase from snake venom and its anticancer potential. Toxicon. 2018;144:7-13.

6. Costa TR, Amstalden MK, Ribeiro DL, Menaldo DL, Sartim MA, Aissa $A F$, et al. CR-LAAO causes genotoxic damage in HepG2 tumor cells by oxidative stress. Toxicology. 2018;404-405:42-8.

7. Ferreira AS, Barraviera B, Barraviera SR, Abbade LP, Caramori CA, Ferreira RS Jr. A success in Toxinology translational research in Brazil: bridging the gap. Toxicon. 2013;69:50-4.

8. Pollegioni L, Motta P, Molla G. L-amino acid oxidase as biocatalyst: a dream too far? Appl Microbiol Biotechnol. 2013;97(21):9323-41.

9. Jain D, Kumar S. Snake venom: a potent anticancer agent. Asian Pac J Cancer Prev. 2012;13(10):4855-60. 
10. Anwar SL, Lehmann U. DNA methylation, microRNAs, and their crosstalk as potential biomarkers in hepatocellular carcinoma. World J Gastroenterol. 2014;20(24):7894-913.

11. Raggi C, Invernizzi P. Methylation and liver cancer. Clin Res Hepatol Gastroenterol. 2013;37(6):564-71.

12. Gnoni A, Santini D, Scartozzi M, Russo A, Licchetta A, Palmieri V, et al. Hepatocellular carcinoma treatment over sorafenib: epigenetics, microRNAs and microenvironment. Is there a light at the end of the tunnel? Expert Opin Ther Targets. 2015;19(12):1623-35.

13. Villanueva A, Portela A, Sayols S, Battiston C, Hoshida Y, Mendez-Gonzalez J, et al. DNA methylation-based prognosis and epidrivers in hepatocellular carcinoma. Hepatology. 2015;61(6):1945-56.

14. Son B, Lee S, Youn H, Kim E, Kim W, Youn B. The role of tumor microenvironment in therapeutic resistance. Oncotarget. 2017;8(3):3933-45.

15. Tavora B, Reynolds LE, Batista S, Demircioglu F, Fernandez I, Lechertier T, et al. Endothelial-cell FAK targeting sensitizes tumours to DNA-damaging therapy. Nature. 2014;514:112-6.

16. Xue X, Gao W, Sun B, Xu Y, Han B, Wang F, et al. Vasohibin 2 is transcriptionally activated and promotes angiogenesis in hepatocellular carcinoma. Oncogene. 2013;32(13):1724-34.

17. Carone SEI, Costa TR, Burin SM, Cintra ACO, Zoccal KF, Bianchini FJ, et al. A new l-amino acid oxidase from Bothrops jararacussu snake venom: Isolation, partial characterization, and assessment of pro-apoptotic and antiprotozoal activities. Int J Biol Macromol. 2017;103:25-35.

18. Bordon KCF, Wiezel GA, Cabral H, Arantes EC. Bordonein-L, a new L-amino acid oxidase from Crotalus durissus terrificus snake venom: isolation, preliminary characterization and enzyme stability. J Venom Anim Toxins Incl Trop Dis. 2015;21:26. doi: 10.1186/s40409-015-0025-8.

19. Bal-Price A, Coecke S. Guidance on Good Cell Culture Practice (GCCP). Neuromethods. 2011;56:1-25.

20. Ding Q, He D, He K, Zhang Q, Tang M, Dai J, et al. Downregulation of TRIM21 contributes to hepatocellular carcinoma carcinogenesis and indicates poor prognosis of cancers. Tumour Biol. 2015;36(11):8761-72.

21. Dong C, He M, Ren R, Xie Y, Yuan D, Dang B, et al. Role of the MAPK pathway in the observed bystander effect in lymphocytes co-cultured with macrophages irradiated with gamma-rays or carbon ions. Life Sci. 2015;127:19-25.

22. Gao Z, Wang H, Xiao FJ, Shi XF, Zhang YK, Xu QQ, et al. SIRT1 mediates Sphk1/S1P-induced proliferation and migration of endothelial cells. Int J Biochem Cell Biol. 2016;74:152-60.

23. Ho CS, Yap SH, Phuah NH, In LL, Hasima N. MicroRNAs associated with tumour migration, invasion and angiogenic properties in A549 and SKLu1 human lung adenocarcinoma cells. Lung Cancer. 2014;83(2):154-62.

24. Mosmann T. Rapid colorimetric assay for cellular growth and survival: application to proliferation and cytotoxicity assays. J Immunol Methods. 1983;65:55-63.

25. Shaddox LM, Mullersman AF, Huang H, Wallet SM, Langaee T, Aukhil I. Epigenetic regulation of inflammation in localized aggressive periodontitis. Clin Epigenetics. 2017;9:94

26. Campos K, Franscisconi CF, Okehie V, de Souza LC, Trombone AP, Letra $A$, et al. FOXP3 DNA methylation levels as a potential biomarker in the development of periapical lesions. J Endod. 2015;41(2):212-8.

27. Costa S, Pereira NB, Pereira K, Campos K, de Castro WH, Diniz MG, et al. DNA methylation pattern of apoptosis-related genes in ameloblastoma. Oral Dis. 2017;23(6):779-83.

28. Karatzas PS, Mantzaris GJ, Safioleas M, Gazouli M. DNA methylation profile of genes involved in inflammation and autoimmunity in inflammatory bowel disease. Medicine (Baltimore). 2014;93(28):e309.

29. Calderon LA, Sobrinho JC, Zaqueo KD, de Moura AA, Grabner AN, Mazzi $M V$, et al. Antitumoral activity of snake venom proteins: new trends in cancer therapy. Biomed Res Int. 2014;2014:203639.

30. Liu CC, Yang H, Zhang LL, Zhang Q, Chen B, Wang Y. Biotoxins for cancer therapy. Asian Pac J Cancer Prev. 2014;15(12):4753-8.

31. Costa TR, Burin SM, Menaldo DL, de Castro FA, Sampaio SV. Snake venom L-amino acid oxidases: an overview on their antitumor effects. J Venom Anim Toxins Incl Trop Dis. 2014;20(1):20-3.
32. Abdelkafi-Koubaa Z, Aissa I, Morjen M, Kharrat N, El Ayeb M, Gargouri $Y$, et al. Interaction of a snake venom L-amino acid oxidase with different cell types membrane. Int J Biol Macromol. 2016;82:757-64.

33. Li Lee M, Chung I, Yee Fung S, Kanthimathi MS, Hong Tan N. Antiproliferative activity of king cobra (Ophiophagus hannah) venom L-amino acid oxidase. Basic Clin Pharmacol Toxicol. 2014;114(4):336-43.

34. Machado ART, Aissa AF, Ribeiro DL, Hernandes LC, Machado CS, Bianchi MLP, et al. The toxin BjussuLAAO-Il induces oxidative stress and DNA damage, upregulates the inflammatory cytokine genes TNF and IL6, and downregulates the apoptotic-related genes BAX, BCL2 and RELA in human Caco-2 cells. Int J Biol Macromol. 2018;109:212-9.

35. Burin SM, Ghisla S, Ouchida AT, Aissa AF, Coelho MG, Costa TR, et al. CR-LAAO antileukemic effect against $\mathrm{Bcr}-\mathrm{Abl}(+)$ cells is mediated by apoptosis and hydrogen peroxide. Int J Biol Macromol. 2016;86:309-20.

36. Wu X, Zhao B, Cheng Y, Yang Y, Huang C, Meng X, et al. Melittin induces PTCH1 expression by down-regulating MeCP2 in human hepatocellular carcinoma SMMC-7721 cells. Toxicol Appl Pharmacol. 2015;288(1):74-83.

37. Yang F, Yang Y, Wang Y, Yang F, Li CL, Wang XL, et al. Selective class I histone deacetylase inhibitors suppress persistent spontaneous nociception and thermal hypersensitivity in a rat model of bee venom-induced inflammatory pain. Sheng Li Xue Bao. 2015;67(5):447-54.

38. Eyvani H, Moghaddaskho F, Kabuli M, Zekri A, Momeny M, TavakkolyBazzaz J, et al. Arsenic trioxide induces cell cycle arrest and alters DNA methylation patterns of cell cycle regulatory genes in colorectal cancer cells. Life Sci. 2016;167:67-7.

39. Nagata M, Kurita H, Uematsu K, Ogawa S, Takahashi K, Hoshina H, et al. Diagnostic value of cyclin-dependent kinase/cyclin-dependent kinase inhibitor expression ratios as biomarkers of locoregional and hematogenous dissemination risks in oral squamous cell carcinoma. Mol Clin Oncol. 2015;3(5):1007-13.

40. Obata Y, Furusawa Y, Endo TA, Sharif J, Takahashi D, Atarashi K, et al. The epigenetic regulator Uhrf1 facilitates the proliferation and maturation of colonic regulatory T cells. Nat Immunol. 2014;15(6):571-9.

41. Jiang Y, Chen J, Tong J, Chen T. Trichloroethylene-induced gene expression and DNA methylation changes in B6C3F1 mouse liver. PLoS One. 2014;9(12):e116179.

42. Nishida N, Goel A. Genetic and epigenetic signatures in human hepatocellular carcinoma: a systematic review. Curr Genomics. 2011;12(2):130-7.

43. Tahara T, Shibata T, Nakamura M, Yamashita H, Yoshioka D, Okubo M, et al. Association between cyclin D1 polymorphism with CpG island promoter methylation status of tumor suppressor genes in gastric cancer. Dig Dis Sci. 2010;55(12):3449-57.

44. Dragoj M, Milosevic Z, Bankovic J, Dinic J, Pesic M, Tanic N, et al. Association of CCND1 overexpression with KRAS and PTEN alterations in specific subtypes of non-small cell lung carcinoma and its influence on patients" outcome. Tumour Biol. 2015;36(11):8773-80.

45. Lin B, Zhou X, Lin S, Wang X, Zhang M, Cao B, et al. Epigenetic silencing of PRSS3 provides growth and metastasis advantage for human hepatocellular carcinoma. J Mol Med (Berl). 2017;95(11):1237-49.

46. Perugini M, larossi DG, Kok CH, Cummings N, Diakiw SM, Brown AL, et al. GADD45A methylation predicts poor overall survival in acute myeloid leukemia and is associated with IDH1/2 and DNMT3A mutations. Leukemia. 2013;27(7):1588-92.

47. Han Y, Zhao H, Jiang Q, Gao H, Wang C. Chemopreventive mechanism of polypeptides from Chlamy Farreri (PCF) against UVB-induced malignant transformation of HaCaT cells. Mutagenesis. 2015;30(2):287-96.

48. Li Z, Gu TP, Weber AR, Shen JZ, Li BZ, Xie ZG, et al. Gadd45a promotes DNA demethylation through TDG. Nucleic Acids Res. 2015;43(8):3986-97.

49. Reis IM, Ramachandran K, Speer C, Gordian E, Singal R. Serum GADD45a methylation is a useful biomarker to distinguish benign vs malignant prostate disease. Br J Cancer. 2015;113(3):460-8.

50. Abreu C, Moreno P, Palacios F, Borge M, Morande P, Landoni Al, et al. Methylation status regulates lipoprotein lipase expression in chronic lymphocytic leukemia. Leuk Lymphoma. 2013;54(8):1844-8. 
51. Yuan L, Chan GC, Fung KL, Chim CS. RANKL expression in myeloma cells is regulated by a network involving RANKL promoter methylation, DNMT1, microRNA and TNFalpha in the microenvironment. Biochim Biophys Acta. 2014;1843(9):1834-8.
52. Xie CR, Sun H, Wang FQ, Li Z, Yin YR, Fang QL, et al. Integrated analysis of gene expression and DNA methylation changes induced by hepatocyte growth factor in human hepatocytes. Mol Med Rep. 2015;12(3):4250-8. 